\title{
PERIODIC BOUNDARY VALUE PROBLEMS OF FIRST AND SECOND ORDER DIFFERENTIAL EQUATIONS
}

\author{
V. Lakshmikantham \\ Department of Applied Mathematics \\ Florida Institute of Technology \\ Melbourne, FL 32901
}

\begin{abstract}
AMS Subject Classification: 34B15, 34C25.
\end{abstract}
Key Words: periodic boundary value problems.

\section{INTRODUCTION.}

Recently the method of upper and lower solutions coupled with Lyapunov Schmidt method and monotone iterative technique has been employed fruitfully to prove theoretical as well as constructive existence results relative to periodic boundary value problems, among others, of first and second order differential equations [2-9], namely,

$$
\begin{gathered}
u^{\prime}=f(t, u), u(0)=u(2 \pi), \\
-u^{\prime \prime}=f(t, u), u(0)=u(2 \pi), u^{\prime}(0)=u^{\prime}(2 \pi) .
\end{gathered}
$$

These considerations crucially depend upon lower and upper solutions $\alpha, \beta$ satisfying the relations

$$
\begin{gathered}
\alpha \leq \beta, \alpha(0) \leq \alpha(2 \pi), \beta(0) \geq \beta(2 \pi), \text { and } \\
\alpha^{\prime}(0) \geq \alpha^{\prime}(2 \pi), \beta^{\prime}(0) \leq \beta^{\prime}(2 \pi),
\end{gathered}
$$

in addition to other assumptions. The problem of proving the existence results when some or all of the foregoing relations are violated is an interesting and important question. In this paper, we shall discuss some known results and raise some open questions.

\footnotetext{
${ }^{0}$ Received November 1988, Revised: August 1989
} 


\section{AN ABSTRACT EXISTENCE RESULT AT RESONANCE.}

Let $E$ be a real Hilbert space. Consider the nonlinear operator equation

$$
L u=N u
$$

where $L: D(L) \subset E \rightarrow E$ is a linear operator and $N: D(N) \subset E \rightarrow E$ is a nonlinear operator with $D(L) \cap D(N) \neq \phi$. Suppose that $P: E \rightarrow E_{0}$ is the projection operator and $H: E_{1} \rightarrow E$ the partial inverse of $L$ on $E_{1}$. Then it is well known that the problem (1) is equivalent to the coupled system of operator equations

$$
\begin{aligned}
u_{1} & =H(1-P) N\left(u_{0}+u_{1}\right), \\
0 & =P N\left(u_{0}+u_{1}\right) .
\end{aligned}
$$

Concerning the problem (1), the following result is known [1].

Theorem 1. Suppose that

i. $\|N u\| \leq J_{0}, u \in D(N)$,

ii. there exist $r_{0}, R_{0}>0$ such that $\left[N\left(u_{0}+u_{1}\right), u_{0}\right] \leq 0$ or $\leq 0$ whenever $\left\|u_{0}\right\|=R_{0}$ and $\left\|u_{1}\right\| \leq r_{0}$ where $u_{0} \in E_{0}$ and $u_{1} \in E_{1}$.

Then the problem (1) admits at least one solution.

We shall employ this result in our discussion.

\section{FIRST ORDER PERIODIC BOUNDARY VALUE PROBLEMS.}

Let us consider the PBVP

$$
u^{\prime}=f(t, u), u(0)=u(2 \pi),
$$

where $f \in C[[0,2 \pi] \times R, R]$. As an application of Theorem 1, we can prove the following result.

Theorem 2. Assume that $\alpha, \beta \in C^{1}[[0,2 \pi], R], \alpha^{\prime} \leq f(t, \alpha), \alpha(0) \leq$ $\alpha(2 \pi), \beta^{\prime} \geq f(t, \beta), \beta(0) \geq \beta(2 \pi)$. Then either $\alpha \leq \beta$ or $\alpha \geq \beta$ on $[0,2 \pi]$ implies that there exists a solution $u$ of PBVP 4 which lies between $\alpha, \beta$. 
Proof. The case $\alpha \leq \beta$ is discussed in [4]. We shall indicate the proof of the other case, namely $\beta \leq \alpha$ on $[0,2 \pi]$. We consider the modified problem

$$
u^{\prime}=F(t, u), u(0)=u(2 \pi),
$$

where $F(t, u)=f(t, p(t, u))+r(t, u), p(t, u)=\max \{\beta, \min \{u, \alpha\}\}$, and

$$
r(t, u)=\left\{\begin{array}{lll}
\frac{u-\alpha}{1+u^{2}} & \text { if } u>\alpha, \\
0 & \text { if } \beta \leq u \leq \alpha, \\
\frac{u-\beta}{1+u^{2}} & \text { if } u \leq \beta .
\end{array}\right.
$$

Clearly $|F(t, u)| \leq J$ on $[0,2 \pi] \times R$, where $J$ depends only on $\beta, \alpha$ and $F$ is continuous. We let $E=L_{2}[0,2 \pi], L u=u^{\prime}, D(L)=\{u \in E: u$ is absolutely continuous, $u^{\prime} \in E$, and $\left.u(0)=u(2 \pi)\right\}$ and $N$ be the operator generated by $F$. Then the PBVP (5) is equivalent to the operator equation (1). We note $E_{0}=N(L)$ consists of constant functions and hence $E_{1}$, where $E=E_{0} \oplus E_{1}$ is the class of all functions whose average is zero. The projection operator $P$ can be defined by $P u=\int_{0}^{2 \pi} u(s) d s$. It is easily seen that the partial inverse operator $H$ also exists and the assumptions required by Theorem 1 are satisfied. Since $F$ is bounded, we can find a constant $A$ which depends on $\alpha, \beta$ such that any solution $u_{1}$ of (2) satisfies the estimate $\left|u_{1}(t)\right| \leq A$ on $[0,2 \pi]$. Hence by Theorem 1 , it is enough to find an $R_{0}>0$ such that

$$
<N\left(u_{0}+u_{1}\right), u_{0}>\geq 0 \text { or } \leq 0,
$$

for all $u_{0}$ and $u_{1}$ such that $\left|u_{0}\right|=r_{0}$ and $\left|u_{1}(t)\right| \leq A$ on $[0,2 \pi]$. Since $u_{0} \in E_{0}$ and $E_{0}=<1>$, the relation (6) with $\geq 0$ is equivalent to

$$
\int_{0}^{2 \pi} F\left(s, R_{0}+u_{1}(s)\right) d s \geq 0 \text { and } \int_{0}^{2 \pi} F\left(s,-R_{0}+u_{1}(s)\right) d s \leq 0 .
$$

(We would like to point out that (6) with $\leq 0$ is utilized in proving the theorem when $\alpha \leq \beta$.) Let us choose $R_{0}>0$ large enough so that we have $R_{0}+u_{1}(t)>\max \{\alpha(t)\}$ and $-R_{0}+u_{1}(t)<\min \{\beta(t)\}$. This choice of $R_{0}$, in view of the definition of $F$, reduces the inequalities (7) to $\int_{0}^{2 \pi} f(s, \alpha(s)) d s \geq$ 0 and $\int_{0}^{2 \pi} f(s, \beta(s)) d s \leq 0$. Since the boundary conditions $\alpha(0) \leq \alpha(2 \pi)$ and $\beta(0) \geq \beta(2 \pi)$ imply the foregoing relations, the PBVP (5) admits at least one solution $u$ by Theorem 1 . 
We shall now show that $\beta \leq u \leq \alpha$ on $[0,2 \pi]$, in which case, $u$ will actually be a solution of the PBVP (4). We shall only prove $\beta \leq u$ since proving $u \leq \alpha$ is similar. Suppose that $\beta \leq u$ on $[0,2 \pi]$ is not true. Then there exists a $t_{0} \in[0,2 \pi]$ and an $\epsilon>0$ such that $\beta\left(t_{0}\right)=u\left(t_{0}\right)+\epsilon$ and $\beta(t) \leq u(t)+\epsilon, t \in[0,2 \pi]$. If $t_{0} \in[0,2 \pi)$, we have $\beta^{\prime}\left(t_{0}\right) \leq u^{\prime}\left(t_{0}\right)$ and $\beta\left(t_{0}\right)>u\left(t_{0}\right)$ which implies that $p\left(t_{0}, u\left(t_{0}\right)\right)=\beta\left(t_{0}\right)$ and $r\left(t_{0}, u\left(t_{0}\right)\right)=$ $\left[u\left(t_{0}\right)-\beta\left(t_{0}\right)\right] /\left[1+u^{2}\left(t_{0}\right)\right]$. Hence

$$
f\left(t_{0}, \beta\left(t_{0}\right)\right) \leq \beta^{\prime}\left(t_{0}\right) \leq u^{\prime}\left(t_{0}\right)=f\left(t_{0}, \beta\left(t_{0}\right)\right)+\frac{u\left(t_{0}\right)-\beta\left(t_{0}\right)}{1+u^{2}\left(t_{0}\right)}<f\left(t_{0}, \beta\left(t_{0}\right)\right),
$$

which is a contradiction. If $t_{0}=2 \pi, \beta(0) \geq \beta(2 \pi)=u(2 \pi)+\epsilon=u(0)+\epsilon$. Consequently, we obtain $\beta^{\prime}(0) \leq u^{\prime}(0)$ which again leads to a contradiction. Thus we have $\beta \leq u \leq \alpha$ on $[0,2 \pi]$ and this proves the theorem.

Note that when $\alpha, \beta$ violate the boundary conditions $\alpha(0) \leq \alpha(2 \pi)$, $\beta(0) \geq \beta(2 \pi)$, proving Theorem 2 is an open question.

Employing the ideas of Theorem 2 repeatedly, one can prove the following result which gives a criterion for the existence of $k$ solutions where $k$ is any given number.

Theorem 3. Assume that $\alpha_{i} \in C^{1}[[0,2 \pi], R]$, and $\alpha_{1} \leq \alpha_{2} \leq \ldots \leq \alpha_{k+1}$. Suppose further that for $i=1,2, \ldots, k+1$, either $(-1)^{i}\left[\alpha_{i}^{\prime}-f\left(t, \alpha_{i}\right)\right] \leq 0$ and $(-1)^{i}\left[\alpha_{i}(0)-\alpha_{i}(2 \pi)\right] \leq 0$ or $(-1)^{i}\left[\alpha_{i}^{\prime}-f\left(t, \alpha_{i}\right)\right] \geq 0$ and $(-1)^{i}\left[\alpha_{i}(0)-\right.$ $\left.\alpha_{i}(2 \pi)\right] \geq 0$ holds. Then there exist at least $k$ solutions $u_{i}$ for the PBVP (4) such that $\alpha_{i} \leq u_{i} \leq \alpha_{i+1}$ on $[0,2 \pi]$ for $i=1,2, \ldots, k$.

Since constructive methods of proving existence results, which can also provide numerical procedures for the computation of solutions, are of greater value than theoretical results, we shall next consider monotone iterative technique. The results we discuss depend on the following lemmas.

Lemma 1. Let $m \in C^{1}[[0,2 \pi], R]$ and $m^{\prime} \leq-M m-\gamma_{m}$ where

$$
\gamma_{m}=\left\{\begin{array}{lll}
0 & \text { if } & m(0) \leq m(2 \pi), \\
M[m(0)-m(2 \pi)] \frac{e^{2 M \pi}}{e^{2 M \pi}-1} & \text { if } & m(0)>m(2 \pi) .
\end{array}\right.
$$

Then $m(t) \leq 0$ on $[0,2 \pi]$.

Lemma 2. Let $m \in C^{1}[[0,2 \pi], R]$ and $m^{\prime} \geq M m+\gamma_{m}$ where

$$
\gamma_{m}=\left\{\begin{array}{lll}
0 & \text { if } & M(0) \geq m(2 \pi), \\
M[m(2 \pi)-m(0)] \frac{e^{2 M \pi}}{e^{2 M \pi}-1} & \text { if } & m(0)<m(2 \pi) .
\end{array}\right.
$$


Then $m(t) \leq 0$ on $[0,2 \pi]$.

Employing monotone iterative technique, we can prove the following result.

Theorem 4. Let $\alpha, \beta \in C^{1}[[0,2 \pi], R]$ and $\alpha^{\prime} \leq f(t, \alpha)-\gamma_{\alpha^{\prime}}, \beta^{\prime} \geq$ $f(t, \beta)+\gamma_{\beta}$ where, for $M>0$,

$$
\begin{aligned}
& \gamma_{\alpha}=\left\{\begin{array}{lll}
0 & \text { if } & \alpha(0) \leq \alpha(2 \pi), \\
M[\alpha(0)-\alpha(2 \pi)] \frac{e^{2 M \pi}}{e^{2 M \pi}-1} & \text { if } & \alpha(0)>\alpha(2 \pi),
\end{array}\right. \\
& \gamma_{\beta}=\left\{\begin{array}{lll}
0 & \text { if } & \beta(0) \geq \beta(2 \pi), \\
M[\beta(2 \pi)-\beta(0)] \frac{e^{2 M \pi}}{e^{2 M \pi}-1} & \text { if } & \beta(0)<\beta(2 \pi) .
\end{array}\right.
\end{aligned}
$$

Suppose further that

i. if $\alpha \leq \beta$, then $f\left(t, u_{1}\right)-f\left(t, u_{2}\right) \geq-M\left(u_{1}-u_{2}\right)$ whenever $\alpha(t) \leq u_{2} \leq u_{1} \leq \beta(t)$

ii. if $\beta \leq \alpha$, then $f\left(t, u_{1} 0-f\left(t, u_{2}\right) \leq M\left(u_{1}-u_{2}\right)\right.$ whenever $\beta(t) \leq u_{2} \leq u_{1} \leq \alpha(t)$.

Then there exist monotone sequences $\left\{\alpha_{n}\right\},\left\{\beta_{n}\right\}$ such that $\lim _{n \rightarrow \infty} \alpha_{n}(t)=$ $\rho(t), \lim _{n \rightarrow \infty} \beta_{n}(t)=r(t)$ uniformly on $[0,2 \pi]$ and that $\rho, r$ are minimal and maximal solutions of PBVP (4) respectively.

Proof. The case relative to (i) is proved in [5]. Here we shall indicate the proof of (ii). For any $\nu \in[\beta, \alpha]=\{\nu \in C[[0,2 \pi], R] \mid \beta \leq \nu \leq \alpha, t \in$ $[0,2 \pi]\}$, we consider the linear PBVP

$$
u^{\prime}-M u=f(t, \nu(t))-M \nu(t), u(0)=u(2 \pi) .
$$

Setting $\delta=f(t, \nu)-M \nu$, we see that

$$
\begin{aligned}
& u(t)=u(0) e^{M t}+\int_{0}^{t} \delta(s) e^{M(t-s)} d s \text { and } \\
& u(0)=u(2 \pi)=\frac{1}{e^{-2 M \pi}-1} \int_{0}^{2 \pi} \delta(s) e^{-M s} d s,
\end{aligned}
$$

satisfy the PBVP (8). By Lemma 2, uniqueness of solutions of (8) follows. Hence for any $\nu \in[\beta, \alpha]$, we define a mapping $A$ by $A \nu=u$, where $u$ is the unique solution of (8). Then we can show by a repeated application of Lemma 2 that $\beta \leq A \beta, A \alpha \leq \alpha$ and $A$ is monotone nondecreasing on $[\beta, \alpha]$. It then follows that we can define the sequences $\left\{\alpha_{n}\right\},\left\{\beta_{n}\right\}$ such that

$$
\beta_{n}=A \beta_{n-1}, \alpha_{n}=A \alpha_{n-1}
$$


and conclude $\beta \leq \beta_{1} \leq \ldots \leq \beta_{n} \leq \alpha_{n} \leq \ldots \leq \alpha_{1} \leq \alpha$ on $[0,2 \pi]$. The rest of the arguments are similar to the case (i) discussed in [5]. Hence the proof is complete.

A repeated application of the ideas of Theorem 4 yields the following result.

Theorem 5. Assume that $\alpha_{i} \in C^{1}[[0,2 \pi], R], i=1,2, \ldots, k+1$ such that $\alpha_{1} \leq \alpha_{2} \leq \ldots \leq \alpha_{k+1}$. Suppose further that, for $i=1,2, \ldots, k+1$, either

$$
\begin{aligned}
(-1)^{i}\left[\alpha_{i}^{\prime}-f\left(t, \alpha_{i}\right)-\gamma_{\alpha_{i}}\right] & \geq 0 \\
\text { and } & \\
(-1)^{i}\left[f\left(t, u_{1}\right)-f\left(t, u_{2}\right)\right] & \leq M\left(u_{1}-u_{2}\right)
\end{aligned}
$$

whenever $\alpha_{i} \leq u_{2} \leq u_{1} \leq \alpha_{i+1}, i=1,2, \ldots, k$ with

$$
\gamma_{\alpha_{i}}=\left\{\begin{array}{lll}
0 & \text { if } & (-1)^{i}\left[\alpha_{i}(0)-\alpha_{i}(2 \pi)\right] \geq 0 \\
M\left[\alpha_{i}(2 \pi)-\alpha_{i}(0) \frac{e^{2 M \pi}}{e^{2 M \pi}-1}\right. & \text { if } & (-1)^{i}\left[\alpha_{i}(0)-\alpha_{i}(2 \pi)\right]<0
\end{array}\right.
$$

or the same relations in (9) hold with reversed inequalities. Then there exist $2 k$ monotone sequences $\left\{\alpha_{n}^{i}\right\}, i=1,2, \ldots, 2 k$, which converge uniformly to $2 k$ extremal solutions $\left(\rho^{i}, r^{i}\right), i=1,2, \ldots, k$ satisfying $\alpha_{i} \leq \rho^{i} \leq r^{i} \leq \alpha_{i+1}$, $i=1,2, \ldots, k$.

Extension of even the simplest result to the PBVP

$$
u^{\prime}=f\left(t, u, u_{t}\right), u(0)=u(2 \pi), u_{0}=\phi_{0},
$$

with $f\left(t, u_{1}, \phi_{1}\right)-f\left(t, u_{2}, \phi_{2}\right) \geq-M\left(u_{1}-u_{2}\right)-N \int_{-\tau}^{0}\left(\phi_{1}-\phi_{2}\right) d s$ whenever $\alpha(t) \leq u_{2} \leq u_{1} \leq \beta(t), \alpha_{t} \leq \phi_{2} \leq \phi_{1} \leq \beta_{t}, t \in[0,2 \pi]$, where $\alpha, \beta$ are lower and upper solutions such that $\alpha \leq \beta$ seems to be difficult and is open.

\section{SECOND ORDER PERIODIC BOUNDARY VALUE PROBLEMS.}

Consider the PBVP

$$
-u^{\prime \prime}=f(t, u), u(0)=u(2 \pi), u^{\prime}(0)=u^{\prime}(2 \pi),
$$

where $f \in C[[0,2 \pi] \times R, R]$. As an application of Theorem 1 , it is easy to prove the following result. See $[2,3]$. 
Theorem 6. Assume that $\alpha, \beta \in C^{2}[[0,2 \pi], R],-\alpha^{\prime \prime} \leq f(t, \alpha), \alpha(0)=$ $\alpha(2 \pi), \alpha^{\prime}(0) \geq \alpha^{\prime}(2 \pi),-\beta^{\prime \prime} \geq f(t, \beta), \beta(0)=\beta(2 \pi), \beta^{\prime}(0) \leq \beta^{\prime}(2 \pi)$ and $\alpha \leq \beta$ on $[0,2 \pi]$. Then there exists a solution $u$ of (10) such that $\alpha \leq u \leq \beta$ on $[0,2 \pi]$.

We do not know whether Theorem 6 is true when $\beta \leq \alpha$. Using monotone iterative technique we can prove the following result. See [5].

Theorem 7. Assume that $\alpha, \beta \in C^{2}[[0,2 \pi], R],-\alpha^{\prime \prime} \leq f(t, \alpha)-\gamma_{\alpha}$, $\alpha(0)=\alpha(2 \pi),-\beta^{\prime \prime} \geq f(t, \beta)+\gamma_{\beta}, \beta(0)=\beta(2 \pi)$ and $\alpha \leq \beta$ on $[0,2 \pi]$, with

$$
\gamma_{\alpha}=\left\{\begin{array}{lll}
0 & \text { if } & \alpha^{\prime}(0) \geq \alpha^{\prime}(2 \pi), \\
\frac{M\left[\alpha^{\prime}(2 \pi)-\alpha^{\prime}(0)\right]\left(e^{2 M \pi}+1\right)}{2\left(e^{2 M \pi}-1\right)} & \text { if } & \alpha^{\prime}(0)<\alpha^{\prime}(2 \pi),
\end{array}\right.
$$

and

$$
\gamma_{\beta}=\left\{\begin{array}{lll}
0 & \text { if } & \beta^{\prime}(0) \leq \beta^{\prime}(2 \pi), \\
\frac{M\left[\beta^{\prime}(0)-\beta^{\prime}(2 \pi)\right]\left(e^{2 M \pi}+1\right)}{2\left(e^{2 W \pi}-1\right)} & \text { if } & \beta^{\prime}(0)<\beta^{\prime}(2 \pi),
\end{array}\right.
$$

uppose further that $f\left(t, u_{1}\right)-f\left(t, u_{2}\right) \geq-M^{2}\left(u_{1}-u_{2}\right)$, whenever $\alpha(t) \leq$ $u_{2} \leq u_{1} \leq \beta(t)$ with $M>0$. Then there exist monotone sequences $\left\{\alpha_{n}\right\}$, $\left\{\beta_{n}\right\}$ such that $\lim _{n \rightarrow \infty} \alpha_{n}=\rho, \lim _{n \rightarrow \infty} \beta_{n}=r$, uniformly on $[0,2 \pi]$, where $\rho, r$ are minimal and maximal solutions of PBVP (10) respectively.

The proof of Theorem 7 crucially depends on the following Lemma.

Lemma 3. Let $m \in C^{2}[[0,2 \pi], R], m(0)=m(2 \pi)$ and $-m^{\prime \prime} \leq-M^{2} m-$ $\gamma_{m}$ where

$$
\gamma_{m}=\left\{\begin{array}{lll}
0 & \text { if } & m^{\prime}(0) \geq m^{\prime}(2 \pi), \\
\frac{M\left[m^{\prime}(2 \pi)-m^{\prime}(0)\right]\left(e^{2 M \pi}+1\right)}{2\left(e^{2 M \pi}-1\right)} & \text { if } & m^{\prime}(0)<m^{\prime}(2 \pi) .
\end{array}\right.
$$

Then $m(t) \leq 0$ on $[0,2 \pi]$.

We do not know whether a result similar to case (ii) of Theorem 4 is true.

Note: This paper was presented at the conference on "Boundary Value Problems" in Trieste, Italy, in May 1984. The proceedings of this conference have never been published. 


\section{REFERENCES.}

1. Cesari, L., and Kannan, R. (1977) An abstract theorem at resonance, Proc. Amer. Math. Soc., 63: 221-225.

2. Kannan, R., and Lakshmikantham, V. (1982) Periodic solutions of nonlinear boundary value problems, Nonlinear Analysis, 6: 1-10.

3. Kannan, R., and Lakshmikantham, V. (1984) Existence of periodic solutions of nonlinear boundary value problems and the method of upper and lower solutions, Appl. Anal., 17: 103-113.

4. Lakshmikantham, V., and Leela, S. (1983) Existence and monotone method for periodic solutions of first order differential equations, J.M.A.A., 91: 237-243.

5. Lakshmikantham, V., and Leela, S. (1984) Remarks on first and second order periodic boundary value problems, Nonlinear Analysis, 8: 281-287.

6. Leela, S. (1983) Monotone method for second order periodic boundary value problems, Nonlinear Analysis, 7: 349-355.

7. Vatsala, A.S. (1983) On the existence of periodic quasi-solutions for first-order systems, Nonlinear Analysis, 7: 1283-1289.

8. Bebernes, J.W., and Schmitt, K. (1973) Periodic boundary value problems for systems of second order differential equations, Jour. Diff. Equations, 13: 32-49.

9. Lakshmikantham, V., Ladde, G.S., and Vatsala, A.S. Monotone Iterative Technology for Nonlinear Differential Equations, Pitman, Boston, 1985. 


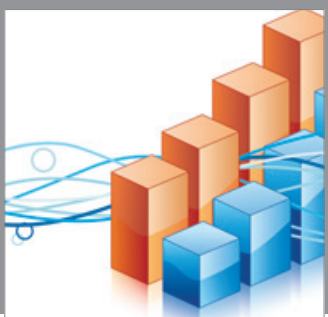

Advances in

Operations Research

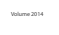

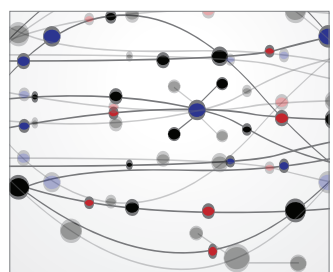

\section{The Scientific} World Journal
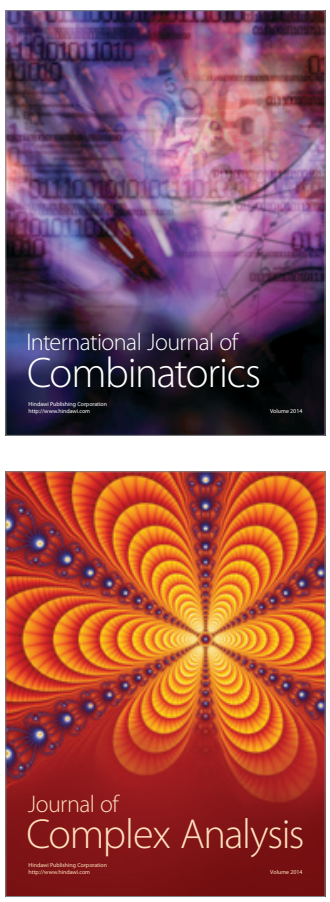

International Journal of

Mathematics and

Mathematical

Sciences
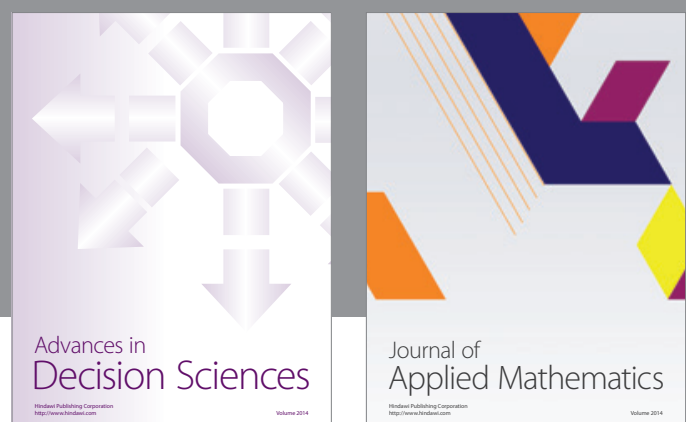

Journal of

Applied Mathematics
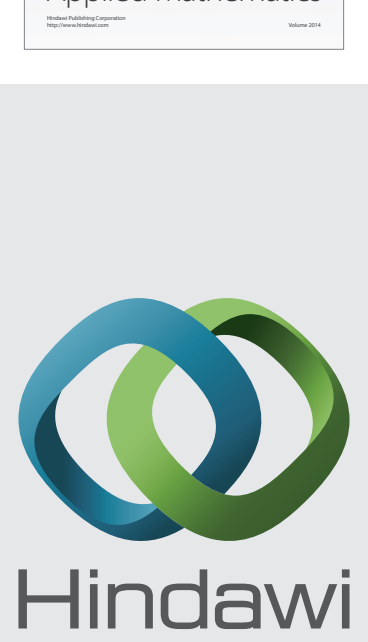

Submit your manuscripts at http://www.hindawi.com
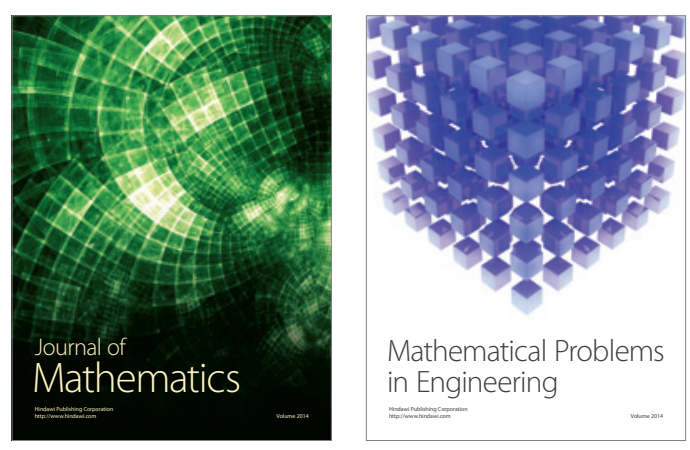

Mathematical Problems in Engineering
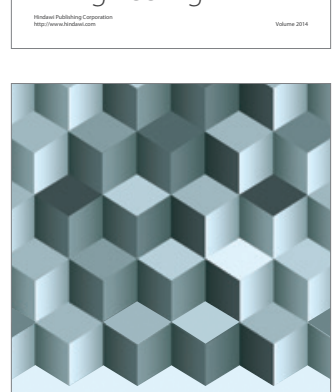

Journal of

Function Spaces
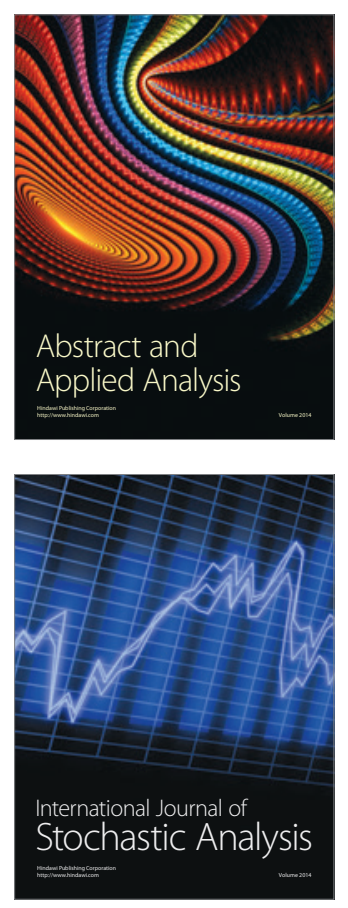

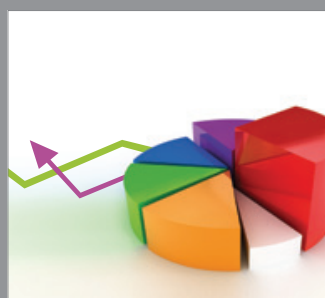

ournal of

Probability and Statistics

Promensencen
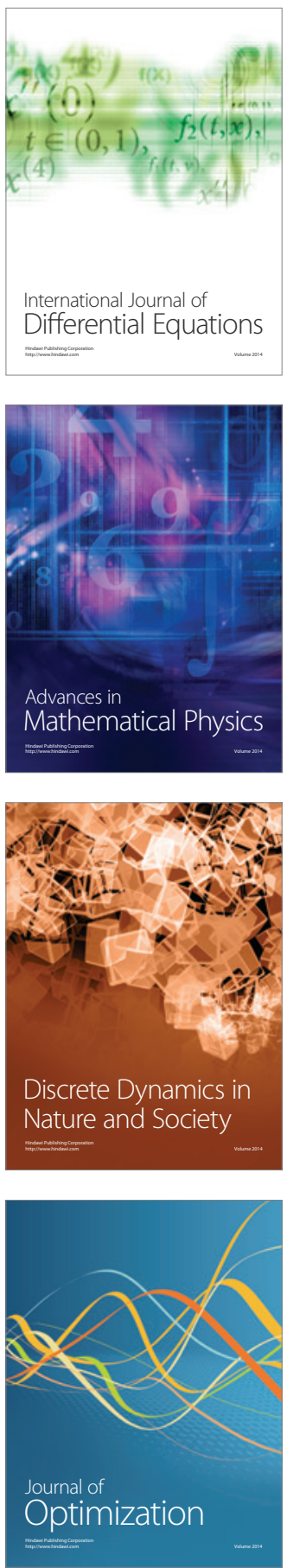